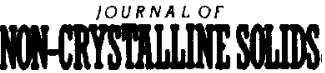

\section{Miscibility in poly(vinylethylene)/poly(isoprene) blends}

\author{
K.J. McGrath*, J.B. Miller, C.M. Roland \\ Chemistry Division, Code 6120, Naval Research Laboratory, Washington, DC 20375-5342, USA
}

\begin{abstract}
Poly(vinylethylene) (PVE) and polyisoprene (PIP) do not chemically interact; nevertheless, miscibility extends to infinite molecular weight of the components, indicating that the interaction parameter for the blend is negative. This inference has been directly demonstrated by small angle neutron scattering, which showed, moreover, that the negative sign has an enthalpic origin. The present solid state nuclear magnetic resonance (NMR) cross-polarization/dipolar dephasing results are consistent with a greater degree of spatial intimacy between PVE and PIP chains in the blend relative to the neat polymers. The greater intimacy suggested by the NMR results is consistent with stronger van der Waals bonding in the blends, and hence the negative mixing enthalpy.
\end{abstract}

\section{Introduction}

It is generally considered that regular mixtures (wherein the components interact only through physical forces and without significant alteration of liquid structure) will have endothermic heats of mixing, and thus rely on combinatorial entropy to effect thermodynamic miscibility. Consequently, the absence of specific interactions will restrict miscibility in van der Waals mixtures to low molecular weight components. Indeed, the interaction parameter, $\chi$, which provides a measure of all noncombinatorial entropy contributions to the free energy, can be measured for a van der Walls blend by determining the molecular weights at which phase segregation occurs [1]. The concept that van der Waals mixtures have positive mixing enthalpies, and hence be miscible only at low component mo-

* Corresponding author.Tel: + 1-202767 3239. Telefax: +12027670594. lecular weights, originates from the assumption that van der Waals bonds between chemically distinct units will be weaker than those associated with like contacts [2]. Hence, the disruption of the like contacts to form unlike ones should be an endothermic process, and mixing is thermodynamically stable only through an entropy gain (i.e., greater positional disorder).

Blends of poly(vinylethylene) (PVE) and 1,4polyisoprene (PIP) have been shown to be thermodynamically miscible by a variety of techniques, including calorimetry [3], proton nuclear magnetic resonance (NMR) [4], spontaneous interdiffusion [5], NMR cross-polarization studies [6], crystallization effects $[7,8]$, orientational coupling ('nematic interaction') [9] and small angle neutron scattering (SANS) $[10,11]$. These seemingly redundant corroborations of miscibility were carried out in part because of the unexpected finding that miscibility in this blend extends to infinite molecular weight of the components $[7,10]$. There are no specific interactions between these components, as 


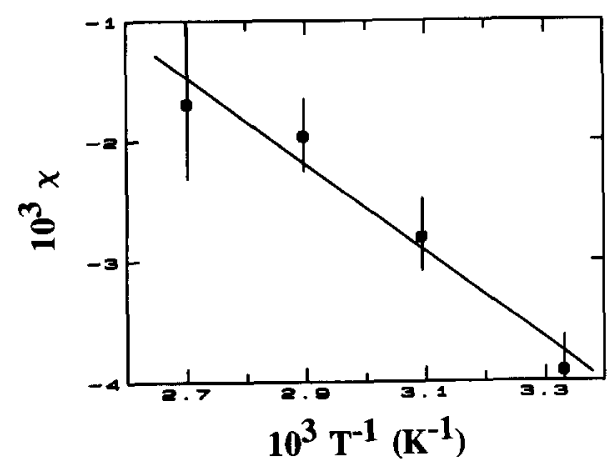

Fig. 1. The temperature dependence of the interaction parameter determined by fitting the measured SANS intensities to the random phase approximation equation [11]. The solid line corresponds to $\chi=8.3 \times 10^{-3}-(3.6 / T)$. The stability of the polymers at the highest temperature is problematical. Omission of the highest temperature datum gives $\chi=1.1 \times 10^{-2}-(4.1 / T)$.

shown by Fourier transform infrared difference spectra [12]. In the absence of chemical interactions, a positive mixing enthalpy is expected, which would limit miscibility to low molecular weights. This contradiction was resolved in part by SANS experiments on blends of PIP and deuterated PVE [11], which demonstrated that the interaction parameter was in fact negative, notwithstanding the absence of specific interactions.

The SANS results [11] clarify how miscibility can extend to very high molecular weights in PVE/PIP blends; however, they do not explain the existence of a negative interaction parameter in a van der Waals mixture. In Fig. 1, the value of $\chi$ obtained by SANS [11] is plotted as a function of temperature. This temperature dependence can be described by the relation

$\chi=8.31 \times 10^{-3}-(3.6 / T)$

If the usual decomposition of the interaction into a temperature-dependent enthalpic part and a temperature-independent entropic contribution is made [13], the conclusion is that the negative sign of $\chi$ has an enthalpic origin. This gives rise to an upper critical solution temperature at $\sim 430 \mathrm{~K}$ (well beyond the decomposition temperatures of the polymers). The question concerning the origin of the negative excess enthalpy remains.
For a van der Waals fluid, the total potential can be accurately represented as the sum of the pair potentials in the system. Thus, for a blend whose components do not specifically interact (i.e., dispersion forces dominate the attractive interactions), the mixing enthalpy can be expressed as

$H^{\mathrm{e}} \sim\left(\varepsilon_{11}+\varepsilon_{22}\right) / 2-\varepsilon_{12}$

For a two-body interaction, the bond energy can be expressed by a series whose leading term is the London formula [14]

$\varepsilon_{12} \sim \alpha_{1} \alpha_{2} / r^{6}+\cdots$

In this equation, $\alpha_{i}$ is the polarizability of the $i$ th chain unit separated by $r$ from the $j$ th unit. Disregarding any dependence of $r$ on composition, the prediction is that the mixing enthalpy must be positive:

$H^{\mathrm{e}} \sim\left[\left(\alpha_{1}^{2} \alpha_{2}^{2}\right) / 2\right]-\alpha_{1} \alpha_{2} \geqslant 0$,

The inference is that the negative value of $\chi$ observed for PVE/PIP mixtures must be due to a composition dependence of $r$; i.e., the intermolecular separations between unlike components in the blend are less than the intermolecular separations in the neat components.

To compare the intermolecular separations in the blend versus the neat liquids, a solid state NMR technique was used. The efficiency of magnetization transfer (cross-polarization) in this experiment has the same $r$ distance dependence seen in Eq. (3) (although in the case of cross-polarization, ' $r$ ' represents internuclear separation rather than distance between polarizable groups). Thus, cross-polarization efficiencies were measured between perdeuterated PVE and hydrogenous PIP, and compared with the efficiency in PVE containing both perdeuterated and hydrogenous chains.

\section{Experimental}

The polymers were a hydrogenous and a perdeuterated PVE (both 97\% 1,2-polybutadiene with weight average degrees of polymerization of 2570 and 3170 , respectively) and 1,4-polyisoprene (70\% cis microstructure, degree of polymeriz- 
ation $=1690$ ). All had narrow molecular weight distributions (polydispersity $\approx 1.2$ ). Two samples were studied, a blend of perdeuterated and hydrogenous PVE and a blend of perdeuterated PVE with hydrogenous PIP. The compositions, approximately $50 \mathrm{wt} \%$ of each component, were adjusted to yield equivalent proton concentration (equal to $0.050 \mathrm{~mol} / \mathrm{cm}^{-3}$ ). Sample weights are accurate to within $\pm 0.5 \%$.

All NMR experiments were run on a Bruker MSL spectrometer with a static magnetic field of 7.04 Tesla. The Hartmann-Hahn technique of cross-polarization [15-17] was employed using $50 \mathrm{kHz}$ radio frequency fields, with proton decoupling during acquisition of the free induction decay, and a magic angle spinning rate of $3.0 \mathrm{kHz}$. The NMR dipolar dephasing technique [16] was used in conjunction with cross-polarization to rapidly dephase (broaden) resonances arising from carbons with directly bonded hydrogen and thereby allow observation of cross-polarized deuterated carbons only. See Refs. $[6,18]$ for further experimental details and a theoretical discussion of cross-relaxation dynamics, respectively.

\section{Results}

Fig. 2 is a plot of the backbone carbon-13 NMR cross-polarization intensities of perdeuterated PVE in the ${ }^{2} \mathrm{HPVE} /{ }^{1} \mathrm{HPVE}$ and ${ }^{2} \mathrm{HPVE} /{ }^{1} \mathrm{HPIP}$ blends as a function of the cross-polarization contact time. All spectra were obtained with a fixed proton dipolar dephasing time of $100 \mu$ s to effect complete broadening of carbons with directly bonded hydrogen, e.g., carbons from the hydrogenous component. The plot contains normalized intensity data obtained at $25 \mathrm{~K}$ below the respective calorimetric glass transition temperatures, $T_{\mathrm{g}}$, of the blends in order to achieve efficient cross polarization. These temperatures correspond to $245 \mathrm{~K}$ for the ${ }^{2} \mathrm{H} \mathrm{PVE} /{ }^{1} \mathrm{H} \mathrm{PVE}$ blend and $220 \mathrm{~K}$ for the ${ }^{2} \mathrm{HPVE} /{ }^{1} \mathrm{H}$ PIP blend. In addition, the ${ }^{2} \mathrm{H} \mathrm{PVE} /{ }^{1} \mathrm{H} \mathrm{PVE}$ blend was run at $220 \mathrm{~K}$ so that the impact of varying temperature (within the ${ }^{2} \mathrm{HPVE} /{ }^{1} \mathrm{H}$ PVE blend) and results at the same temperature (for different blends) could be compared.

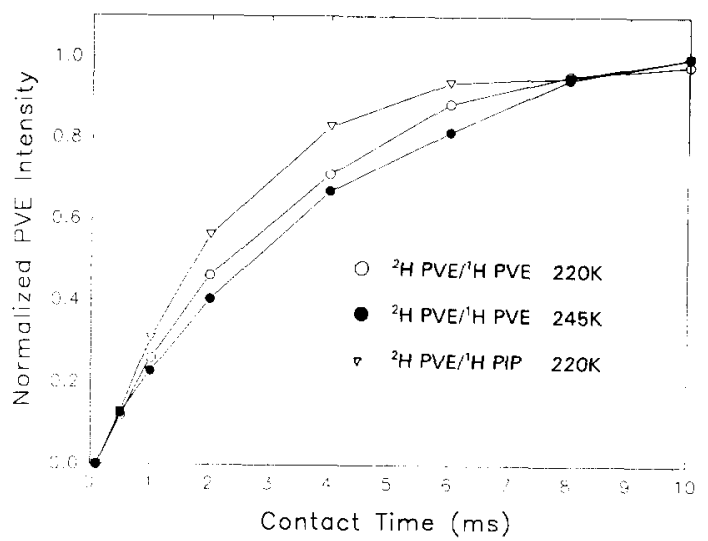

Fig. 2. Plot of deuterated PVE saturated NMR carbon resonance intensity versus cross-polarization contact time with a fixed $100 \mu \mathrm{s}$ dipolar dephasing time. Note that the crossrelaxation time, $T_{\mathrm{LS}}$ for the ${ }^{1} \mathrm{H} \mathrm{PIP} /{ }^{2} \mathrm{PVE}$ blend is significantly shorter $(2.1 \pm 0.1 \mathrm{~ms})$ relative to the ${ }^{1} \mathrm{H} \mathrm{PVE} /{ }^{2} \mathrm{H}$ PVE blend $(3.1 \pm 0.2 \mathrm{~ms}$ at $220 \mathrm{~K}$ and $3.6 \pm 0.2 \mathrm{~ms}$ at $245 \mathrm{~K})$. The lines are drawn as guides for the eye.

The time constant, $T_{\text {IS }}$ associated with crosspolarization of the backbone PVE carbons (Fig. 2) in the ${ }^{2} \mathrm{H} \mathrm{PVE} /{ }^{1} \mathrm{H}$ PIP blend at $220 \mathrm{~K}$ corresponds to $2.1 \pm 0.1 \mathrm{~ms}$. By contrast, the time constant for cross-polarization of backbone carbons in the ${ }^{2} \mathrm{H} \mathrm{PVE} /{ }^{1} \mathrm{H}$ PVE blend at $245 \mathrm{~K}$ was $3.6 \pm 0.2 \mathrm{~ms}$. Virtually the same cross-polarization time constant of $3.1 \pm 0.2 \mathrm{~ms}$ was calculated for this blend at $220 \mathrm{~K}$

Similar determinations of the cross-polarization time constants were made for each of the two downfield unsaturated carbon resonances of perdeuterated PVE in these blends. The results of these calculations are shown in Table 1. In all cases it is observed that the time constant associated with cross-polarization of the various carbon resonances of ${ }^{2} \mathrm{HPVE}$ is significantly shorter in the ${ }^{2} \mathrm{HPVE} /{ }^{1} \mathrm{H}$ PIP blend compared with the ${ }^{2} \mathrm{HPVE} /{ }^{1} \mathrm{H} \mathrm{PVE}$ blend. In addition, the longest time constants are observed for the backbone carbon atoms while the shortest occur in the vinyl carbons of PVE. This result is consistent with the expectation that the deuterated pendant vinyl carbons of PVE are in closer spatial proximity to the hydrogen nuclei of the non-deuterated component. For a given blend, no differences (within experimental error) were observed in the cross relaxation 
Table 1

Cross-relaxation time constants, $T_{\mathrm{IS}}(\mathrm{ms})$, for perdeuterated carbons of PVE in ${ }^{1} \mathrm{H} \mathrm{PVE} /{ }^{2} \mathrm{H} \mathrm{PVE}$ and ${ }^{1} \mathrm{PIP} /{ }^{2} \mathrm{H} \mathrm{PVE}$ Blends

\begin{tabular}{llll}
\hline${ }^{2} \mathrm{H}$ PVE moiety & $\begin{array}{l}{ }^{1} \mathrm{H} \mathrm{PIP} /{ }^{2} \mathrm{H} \mathrm{PVE} \\
(220 \mathrm{~K})\end{array}$ & $\begin{array}{l}{ }^{1} \mathrm{H} \mathrm{PVE} /{ }^{2} \mathrm{H} \mathrm{PVE} \\
(220 \mathrm{~K})\end{array}$ & $\begin{array}{l}{ }^{1} \mathrm{H} \mathrm{PVE} /{ }^{2} \mathrm{H} \mathrm{PVE} \\
(245 \mathrm{~K})\end{array}$ \\
\hline$-\mathrm{CD}_{2}-\mathrm{CD}-$ & $2.1 \pm 0.1$ & $3.1 \pm 0.2$ & $3.6 \pm 0.2$ \\
$-\mathrm{CD}_{-}$ & $1.8 \pm 0.2$ & $2.4 \pm 0.2$ & $2.5 \pm 0.2$ \\
$-\mathrm{CD}_{2}$ & $1.5 \pm 0.2$ & $2.6 \pm 0.3$ & $2.9 \pm 0.3$ \\
\hline
\end{tabular}

time constants of the two vinyl carbons of ${ }^{2} \mathrm{H}$ PVE (Table 1).

\section{Discussion}

The rate of cross-polarization, $T_{\mathrm{IS}}^{-1}$, for a higheffective-field spin lock NMR experiment such as was used in our investigation is given by [18]

$T_{\mathrm{IS}}^{-1}=K M_{2, \mathrm{Sl}} J_{\mathrm{x}}\left(\Delta \omega_{\mathrm{eff}}\right)$,

where $K$ relates to the resonance offset $(K \approx 1.0$ for our experimental procedure), $M_{2, \text { sI }}$ is the second moment (or mean square linewidth) of the carbon nuclei dipolar coupled to the proton nuclei and $J_{x}\left(\Delta \omega_{\text {eff }}\right)$ is the cross-polarization spectral density function of the proton spins (see above). The second moment is directly proportional to $r^{-6}$, where $r$ represents the average internuclear separation between carbon and hydrogen [19]. Thus, the cross-polarization rate of perdeuterated PVE in this investigation is strongly governed by the distance separating the perdeuterated PVE carbons and the hydrogens of the hydrogenous component (e.g., ${ }^{1} \mathrm{H}$ PVE of ${ }^{1} \mathrm{H}$ PIP).

The rate of cross-polarization of perdeuterated PVE is significantly higher for all carbons of PVE when blended with PIP. For example, $T_{\text {IS }}$ (Table 1) for the downfield vinyl carbon of ${ }^{2} \mathrm{H} \mathrm{PVE}$ is $25 \%$ shorter in the PVE/PIP blend ( $220 \mathrm{~K})$ compared with the PVE/PVE blend ( 220 and $245 \mathrm{~K}$ ). A decrease in $T_{\text {IS }}$ of this magnitude would require an approximate $7 \%$ densification in the PVE/PVE blend; the measured densification is only $0.1 \%$ [20]. Even larger differences in the cross-polarization time constants are observed for the other carbons of PVE. Thus, the large decrease in $T_{\text {IS }}$ for all PVE carbons of the PVE/PIP blend compared with the same carbons of the PVE/PVE blend is consistent with closer spatial proximity of the PVE and PIP components in the blend. This finding is consistent with the negative mixing enthalpy determined from SANS (Fig. 1).

A previous NMR investigation of PVE/PIP blends [4] demonstrated the existence of 'dynamical heterogeneity' of the components; i.e., above the glass transition temperature PVE and PIP exhibit different segmental dynamics. This difference in timescale for their motions could conceivably modulate the dipolar interactions differently in PVE/PIP versus PVE/PVE blends, and thus conceivably give rise to the observed differences in cross-relaxation time constants. However, all crosspolarization experiments were performed at 25 and $50 \mathrm{~K}$ below the calorimetric glass transition temperatures of the blends, where large scale molecular motion (and therefore any consequences of dynamical heterogeneity) is absent. It is therefore unlikely that the differences in PVE and PIP mobility known to exist above the glass transition signifcantly influence the dynamics of cross-polarization at the temperatures used in this investigation. The equivalence (within experimental error) of the three cross-relaxation time constants for the PVE/PVE blend at 220 and $245 \mathrm{~K}$ (Table 1) also evidence the absence of motional effects on the efficiency of cross-polarization at these temperatures.

It is also possible that a region of high proton spin density (e.g., the methyl moiety of PIP) could account for the increased rate of cross-polarization of deuterated PVE carbons when ${ }^{1} \mathrm{H}$ PIP is present. If this were the case, a rapid buildup to the ${ }^{2} \mathrm{H}$ PVE carbon intensity would be observed in the blend containing ${ }^{1} \mathrm{H}$ PIP, but a lower normalized intensity of the ${ }^{2} \mathrm{H}$ PVE carbons would be expected (relative to the ${ }^{2} \mathrm{H}$ PVE $/{ }^{1} \mathrm{H}$ PVE blend). However, the nor- 
malized intensities of cross-polarized ${ }^{2} \mathrm{H}$ PVE carbons at a given contact time were not significantly different when the relative quantities of components were taken into account. This result suggests that the increased rate of cross-polarization of the ${ }^{2} \mathrm{H}$ PVE carbons in the presence of ${ }^{1} \mathrm{H}$ PIP is not simply attributable to regions of higher proton spin density. However, the decreased normalized ${ }^{13} \mathrm{C}$ intensity of PVE which would be expected for cross-polarization from a high proton spin density region may not be of sufficient magnitude to be detected in our experiments.

Finally, we consider the consequences of the spectral density term, $J_{x}\left(\Delta \omega_{\text {eff }}\right)$, in Eq (5) on the measured $T_{\text {IS }}$. For a high effective field spin lock experiment in which the deviation from the Hartmann-Hahn match is negligible, this spectral density function can be approximated as being directly proportional to the correlation time of the proton-proton dipolar fluctuations. This dipolar fluctuation time will in turn be largely dependent upon the local proton density since all experiments were carried out well below the $T_{\mathrm{g}}$ of the blends, where molecular motions will not significantly modulate the homonuclear dipolar interaction among protons. Both the PVE/PIP and PVE/PVE blends were prepared to have equal bulk hydrogen concentrations. Nonetheless, the local proton density of pure PIP is approximately $8 \%$ higher than that of pure PVE. Thus, stronger proton dipole-dipole couplings and shorter proton dipolar fluctuation times are expected in the PVE/PIP blend relative to the PVE/PVE blend. A shorter proton fluctuation time would have the effect reducing the spectral density function, $J_{x}$, and thus reducing the rate of crosspolarization in the PVE/PIP blend (relative to the PVE/PVE blend). Since a higher rate of cross polarization is observed in the PVE/PIP blend, the spectral density term $J_{\mathrm{x}}$ in $\mathrm{Eq}(5)$ cannot account for the observed diminution of $T_{\text {IS }}$ in this blend.

\section{Conclusion}

The ${ }^{13} \mathrm{C}$ NMR cross-polarization time constants measured in a blend of ${ }^{2} \mathrm{H} \mathrm{PVE} /{ }^{1} \mathrm{H}$ PIP are signifi cantly smaller than for a blend of ${ }^{2} \mathrm{HPVE} /$ ${ }^{1} \mathrm{H}$ PVE. This result is consistent with closer spatial proximity of chains in the PVE/PIP blend relative to the neat components. This greater spatial; intimacy accounts for the anomalous negative excess enthalpy in PVE/PIP blends.

This work was supported by the Office of Naval Research. The authors thank Dr. A. N. Garroway for helpful comments on the manuscript.

\section{References}

[1] C.A. Trask and C.M. Roland, Polym. Commun. 29 (1988) 332.

[2] J.H. Hildebrand and R.L. Scott, Regular Solutions (Prentice-Hall, Endlgewood Cliffts, N.J. 1962).

[3] G.M. Bartenev and G.S. Kongarov, Rubber. Chem. Technol. 36 (1963) 668.

[4] J.B. Miller, K.J. McGrath, C.M. Roland, C.A. Trask and A.N. Garroway, Macromolecules 23 (1990) 4543.

[5] C.M. Roland, Macromolecules 20 (1987) 2557.

[6] C.M. Roland, J.B. Miller and K.J. McGrath, Macromolecules 26 (1993) 4967.

[7] I.S. Zemel and C.M. Roland, Polymer 33 (1992) 3427.

[8] D.W. Tomlin and C.M. Roland, Polymer 34 (1993) 2665.

[9] I.S. Zemel and C.M. Roland, Polymer 33 (1992) 4522.

[10] C.M. Roland and C.A. Trask, Macromolecules 22 (1989) 256.

[11] D.W. Tomlin and C.M. Roland, Macromolecules 25 (1992) 2994.

[12] C.M. Roland, J. Poly. Sci. Poly. Phys. Ed. 26 (1988) 839.

[13] P.J. Flory and W.R. Krigbaum, Ann. Rev. Phys. Chem. 2 (1951) 383.

[14] J.O. Hirschfelder and C.F. Curtiss R.B. Bird, Molecular Theory of Gases and Liquids (Wiley, New York, 1967) Ch. 13.

[15] S.R. Hartmann and E.L. Hahn, Phys. Rev, 128 (1962) 2042.

[16] A. Pines, M.G. Gibby and J.S. Waugh, J. Chem Phys. 59 (1973) 569.

[17] J. Schaefer and E.O. Stejskal, J. Am. Chem. Soc. 98 (1976) 1031.

[18] D.E. Demco, J. Tegenfeldt and J.S. Waugh, Phys. Rev. B11 (1975) 4133

[19] C.P. Slichter, Principles of Magnetic Resonance, Springer Series in Solid-State Sciences, Vol. 1, 2nd Ed. (Springer New York, 1980).

[20] J. Roovers and P.M. Toporowski, Macromolecules 25 (1992) 3454 